\title{
Influence of current pulse shape on directly modulated system performance in metro area optical networks
}

\author{
Carmina del Rio Campos ${ }^{\mathrm{a}, *}$, Paloma R. Horche ${ }^{\mathrm{b}, 1}$, Alfredo Martin-Minguez ${ }^{\mathrm{b}}$ \\ a Departamento de Ingeniería de Sistemas Electrónicos y Telecomunicación Universidad San Pablo CEU de Madrid, Urbanización Montepríncipe, Boadilla del Monte, Madrid, Spain \\ b Departamento de Tecnología Fotónica, ETSI Telecomunicación-UPM, Ciudad Universitaria s/n, 28040-Madrid, Spain
}

\section{A R T I C L E I N F O}

Keywords:

Adiabatic and transient chirp

Chromatic dispersion fibers

Directly modulated lasers

Modulation current shape

\begin{abstract}
A B S T R A C T
Due to the fact that a metro network market is very cost sensitive, direct modulated schemes appear attractive. In this paper a CWDM (Coarse Wavelength Division Multiplexing) system is studied in detail by means of an Optical Communication System Design Software; a detailed study of the modulated current shape (exponential, sine and gaussian) for $2.5 \mathrm{~Gb} / \mathrm{s}$ CWDM Metropolitan Area Networks is performed to evaluate its tolerance to linear impairments such as signal-to-noise-ratio degradation and dispersion. Point-to-point links are investigated and optimum design parameters are obtained. Through extensive sets of simulation results, it is shown that some of these shape pulses are more tolerant to dispersion when compared with conventional gaussian shape pulses. In order to achieve a low Bit Error Rate (BER), different types of optical transmitters are considered including strongly adiabatic and transient chirp dominated Directly Modulated Lasers (DMLs). We have used fibers with different dispersion characteristics, showing that the system performance depends, strongly, on the chosen DML-fiber couple.
\end{abstract}

\section{Introduction}

The proliferation of high-bandwidth applications has motivated a growing interest, between network providers, on upgrading networks to deliver broadband services to homes and small businesses. There has to be a great efficiency between the total cost of the infrastructures and the services that can be offered to the end users, very sensitive to equipment costs, requiring the use of low-cost optical components. Coarse Wavelength Division Multiplexing (CWDM) is an ideal solution to the tradeoff between cost and capacity. This technology uses all or part of the 1270 to $1610 \mathrm{~nm}$ wavelength fiber range with an optical channel separation of about $20 \mathrm{~nm}$. This channel separation allows the use of low-cost, not cooled, Directly Modulated Lasers (DMLs). The broadening of the transmitted pulse due to the propagation in a dispersive media, as the optical fiber is, produces one of the main limitations for the length or the transmission bit rate of an optical communication link. The pulse broadening, after being propagated by a distance through the fiber, is a function of different factors, like the initial shape of the pulse, the chirp associated with and the dispersive characteristics of the optical fiber [1].

In directly modulated systems, the shape of the pulse propagated through the fiber depends, mainly, on the electric pulse applied to the

\footnotetext{
* Corresponding author. Universidad San Pablo CEU de Madrid, Dpto. Ingeniería Sistemas Electrónicos y Telecomunicación, 28660 Boadilla del Monte (Madrid), Spain. E-mail addresses: carmina.eps@ceu.es (C.R. Campos), phorche@tfo.upm.es

(P.R. Horche), alfredo.minguez@tfo.upm.es (A. Martin-Minguez).

1 Member, OSA
}

optical source (modulation current) and the dynamic response of the laser for large-signal inputs. The Non Return to Zero (NRZ) format is most often used in practice for the modulation current of the resulting optical bit stream. Ideally, the NZR electrical pulses are rectangular with abrupt rise and fall edges; in practice, these pulses are nonideal and which rise and fall edges are mathematically modeled with sine, exponential, gaussian, etc expressions.

The frequency chirp for large-signal modulation can be determined from the shape of the modulated signal. If we are interested in minimizing the frequency chirp, it is mandatory to perform a study that involves the output waveforms in order to predict which of them produces the lowest chirp. We can then tailor the current input signal accordingly.

On the other hand, the best part of the theoretical studies related to the transmission of pulses across an optical fiber suppose, for analytical simplicity, is that the generator of electrical pulses provides a gaussian shape in time.

In this work, by software simulations, the consequences of nonideal pulse generator are analyzed. Also, we present results about how to optimize the transmission performance of an optical communication system depending on the modulation current used for the laser. To do this, the shape of the current input signal is modeled according to different mathematical expressions (sine, exponential, gaussian, etc).

Since already it has been mentioned, there are other system elements and parameters affecting the shape of the transmitted optical pulse, mainly the DML output optical power and the dispersive characteristics of the optical fiber. Due to this fact, in this work, we 
have used two types of optical fiber; the already laid and widely deployed, single-mode ITU-T G.652 fiber (SMF) and the ITUT-T G.655 fiber with a negative dispersion sign around the $\mathrm{C}$ band (NZ-DSF); in all simulated cases, a peak power for the " 1 " bit between 0.1 and $10 \mathrm{~mW}$ has been considered.

This paper is organized as follows: Section 2 is dealing with the theoretical background where the subject of this paper is based; Section 3 represents the system model where the different types of fibers, DMLs and modulation current are simulated. In Section 4, we analyze the results obtained with the simulations. Finally, the conclusions are summarized in Section 5.

\section{Simulation methodology}

To analyze a directly modulated WDM system, in order to optimize its transmission performance, a basic link is considered (see Fig. 1). A block diagram of the directly modulated transmitter to be considered is illustrated in Fig. 2.

The injected laser current is given by the expression:

$I(t)=I_{b}+\sum_{k=-\infty}^{\infty} a_{k} I_{p}(t-k T)$

where $I_{b}$ is the bias current, $T$ is the period of the modulation pulse, the sequence of bits transmitted $\left(a_{k}=1(0)\right.$ if a binary one (zero) is transmitted during the $k_{\mathrm{th}}$ time), and $I_{p}(t)$ is the applied current pulse.

In a free-chirp source, the optical power output pulse of the laser, $P(t)$, is given by

$P(t)=\eta_{0} \cdot \frac{h \nu}{q} \cdot \sum_{k=-\infty}^{+\infty} a_{k} I_{p}(t-k T)$

where $\eta_{0}$ is the differential quantum efficiency of the laser, $h \nu$ is the photon energy at the optical frequency $\nu$, and $I_{p}(t)$ is the applied current pulse.

However, expression (2) is not applicable in the case of directly modulated sources where the injected current that modulates the laser, introduces a shift in the emission frequency (chirp frequency). As a consequence, the optical power output pulse is not a linear transformation of the applied current pulse.

The optical power and the chirping response of the semiconductor laser to the current waveform $I(t)$ are determined by means of the largesignal rate equations [2], which describe the interrelationship of the photon density, carrier density, and optical phase within the laser cavity.

Thus, the chirp associated with the output of the laser can be formulated by

$\Delta \nu(t)=\frac{1}{2 \pi} \frac{d \phi}{d t}$

where $\phi$ is the optical phase, which variation with the time depends on the line-width factor or Henry's factor, $\alpha$ [3]:

$\frac{d \phi}{d t}=\frac{1}{2} \alpha\left[\Gamma v_{g} a_{0}\left(n-n_{t}\right)-\frac{1}{\tau_{p}}\right]$

$n_{t}$ represents the carrier density at transparency, $\Gamma$ is the confinement factor, $v_{\mathrm{g}}$ is the group velocity, $a_{0}$ is the gain coefficient, and $\tau_{\mathrm{p}}$ is the

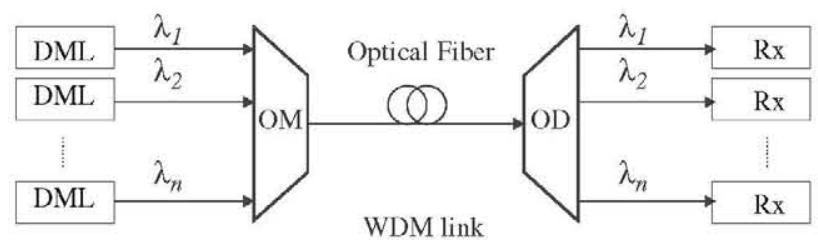

Fig. 1. Block diagram of a CWDM system.

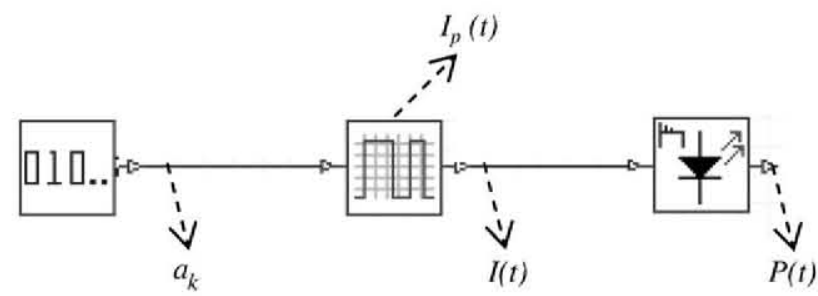

Fig. 2. Directly modulated laser scheme.

photon lifetime. Under this conditions and taking into account that $n_{t} \propto P$, the frequency chirp associated with DML depends on the value taken by the optical power weighted by the parameters $\alpha$ and $\kappa$ according to the equation:

$\Delta v(t)=\frac{\alpha}{4 \pi}\left(\frac{1 d P(t)}{P(t)} d t+\kappa P(t)\right)$

where $k$ is the adiabatic coefficient determined from the parameters: gain compression factor $(\varepsilon)$, differential quantum efficiency $(\eta)$, active layer volume $(V)$, and the mode confinement factor $(\Gamma)$ according to the following equation:

$\kappa=\frac{2 \Gamma \varepsilon}{\eta h \nu V}$

The first term of Eq. (5), transient chirp, produces variations in the pulse width, while the second term, adiabatic chirp, produces a different frequency variation between the " 1 " and " 0 " bits causing a shift in time between the levels corresponding to " 1 " and " 0 " bits when the pulses go through a dispersive media, as the optical fiber.

With Eq. (5), the frequency chirp for large-signal modulation can be determined directly from the shape of the modulated signal. This equation can be used to predict which output waveforms produce the lowest chirp and we can tailor the current input signal accordingly.

Nevertheless, when an optical pulse is transmitted through a dispersive media, like an optical fiber, the intensity and the shape of the optical signal at the output of the optical fiber, due to the waveform $I(t)$, are related with the dispersive characteristics of the optical fiber.

Summarizing, the pulse shape is a function of different factors: the initial pulse shape, the chirp associated with this pulse from the DML and the dispersive characteristics of the optical fiber. If we suppose an initial pulse with a gaussian form and considering no-linear effects in the fiber to be negligible, the shape of the pulse, after a distance $z$, is given by the equation governing the evolution of the complex amplitude of the field envelope along the fiber, $A(z, t)[1]$ :

$A(\xi, t)=\frac{A_{0}}{\sqrt{b_{f}}} \exp \left[-\frac{\left(1+i C_{1}\right) t^{2}}{2 T_{0} b_{f}^{2}}+\frac{i}{2} \tan ^{-1}\left(\frac{\xi}{1+C \xi}\right)\right]$

where $A_{0}$ is the peak amplitude, $T_{0}$ represents the half-width of the pulse at $1 /$ e power point and $b_{f}$ is the pulse broadening factor. The parameter $C$ in Eq. (7) takes into account the frequency chirp of the pulse at $z=0$ (DML chirp) and $C_{1}$ represents the value of the chirp associated with the pulse after a distance $z$. Expression (7) is depending on the normalized distance $\left(\xi=z / L_{D}\right)$, where $L_{D}$ is the dispersion length, defined as $L_{D}=\left(T_{0}\right)^{2} /\left|\beta_{2}\right|$ where $\beta_{2}$ is the Group Velocity Dispersion (GVD) and it is related to the dispersion parameter, $D$, of the fiber. 
Therefore, when a pulse with an initial chirp $C$, crosses a distance $\xi$ in a dispersive media, the chirp will acquire a new value given by $C_{1}$ and the pulse will be broadened by a factor represented by $b_{f}$.

$b_{f}(\xi)=\left[(1+s C \xi)^{2}+\xi^{2}\right]^{1 / 2} \quad C_{1}(\xi)=C+\left(s\left(1+C^{2}\right) \xi\right.$

where $s$ is the sign of $\beta_{2}$ with a value of $+1-1$.

As expected, the shape of the signal at the output of the optical fiber is affected by the sign of both $\beta_{2}$ (fiber parameter) and $C$ (transmitter parameter). For this reason, in this work, we have simulated two fibers with opposite sign in its dispersion coefficient and two DMLs with different characteristics.

\section{System model}

Fig. 1 shows the basic WDM link implemented in our simulations. In this work we are specifically interested in how a system is impacted by the pulse shape of the modulation current. But our final purpose is to optimize a CWDM system for use in a metropolitan area optical network. In the case of a directly modulated laser, the system behaviour is very sensitive to the spectral response of WDM components, such as multiplexers, demultiplexers and filters because the shape of the pulse, before it reaches the receiver, is changing as a result of their transmission across these components.

For this reason, we have simulated the WDM link shown in Fig. 1 including the WDM components. Nevertheless, only the results obtained for one channel (i.e. the channel allocated at $1551 \mathrm{~nm}$ ) will be presented in this paper.

\subsection{Optical fibers}

As already mentioned, the chromatic dispersion coefficient, both in absolute terms and in sign, is one of the most influential fiber parameters on the transmission performance when a DML is used as a transmitter. We have used two types of optical fiber; the already laid and widely deployed, single-mode ITU-T G.652 fiber (SMF) and the ITUT-T G.655 fiber (N-DSF). It is well known that the SMF fiber dispersion coefficient is positive in the telecommunication band spectrum, from $\mathrm{O}$ to $\mathrm{L}$ band, and the dispersion coefficient sign of the $\mathrm{N}$-DSF fiber is negative around $\mathrm{C}$ band. In this work, the same spectral attenuation coefficient of both fibers has been considered whose water peak at $1.38 \mu \mathrm{m}$ is well suppressed. The dispersion slope, effective area and nonlinear index of refraction are compliant with typical conventional G.652 and G.655 fiber recommendations.

\subsection{Transmitter}

The transmitter block diagram implemented in our simulations is shown in Fig. 2. It consists of a bit random generator, which

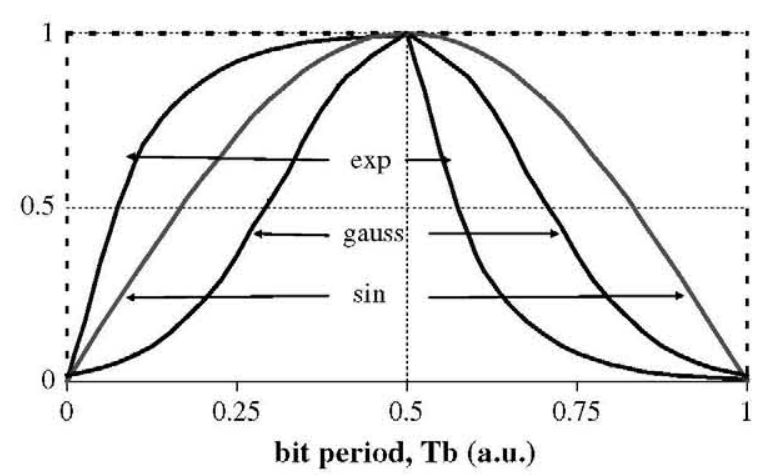

Fig. 3. Exponential, gaussian and sine pulse shape for the laser modulation current. determines the sequence of bits, $a_{k}$, that will be sent to an electric pulse generator, with the NRZ format, which injects a modulation current, $I(t)$ to the laser diode (DML), not cooled. The pulse string was encrypted with a $2^{15}-1$ pseudo-random bit sequence, OC-48 system, at $2.5 \mathrm{Gbit} / \mathrm{s}$.

For our purpose, the leading and tailing edges of the applied current pulse $I_{p}(t)$ have been matched to different forms. The usual non-ideal pulse shapes can be simulated by exponential, sine and gaussian mathematical expressions (see Fig. 3):

$$
\begin{aligned}
& \text { Exponential : } I_{p}(t)=\left\{\begin{array}{l}
1-e-{ }^{\left(t / c_{r}\right)} \rightarrow 0 \leq t \leq t_{1} \\
1 \rightarrow t_{1} \leq t \leq t_{2} \\
e^{-\left(t / c_{f}\right)} \rightarrow t_{2} \leq T_{b}
\end{array}\right. \\
& \text { Gaussian: } I_{p}(t)=\left\{\begin{array}{l}
e-{ }^{\left(t / c_{r}\right)^{2}}-1 \rightarrow 0 \leq t \leq t_{1} \\
1 \rightarrow t_{1} \leq t \leq t_{2} \\
e^{-\left(t / c_{f}\right)^{2}} \rightarrow t_{2} \leq T_{b}
\end{array}\right. \\
& \text { Sine: } \quad I_{p}(t)=\left\{\begin{array}{l}
\sin \left(\pi t / c_{r}\right) \rightarrow 0 \leq t \leq t_{1} \\
1 \rightarrow t_{1} \leq t \leq t_{2} \\
\sin \left(\pi t / c_{f}\right) \rightarrow t_{2} \leq T_{b}
\end{array}\right.
\end{aligned}
$$

where, $c_{r}$ and $c_{f}$ are the rise and fall time coefficients, respectively. $\mathrm{t}_{1}$ and $t_{2}$, together with $c_{r}$ and $c_{f}$, are numerically determined to generate pulses with the exact values of the rise time and fall time parameters, and $T_{b}$ is the bit period. In all simulated cases, fall and rise times are fixed at the half bit period.

To simulate the DML, it has been used to model the "Laser Rate Equations" introduced in [4]. In the simulation, we have chosen values of commercial lasers to generate two types of chirp behaviour: DML-A with a dominant adiabatic chirp and DML-T with a dominant transient chirp. For this, we work with two typical values for the Henry coefficient, $(\alpha)$ [5] and the adiabatic coefficients $(k)$ have been determined from the parameters $\varepsilon$, $\eta$, and $\Gamma$, according to Eq. (6). These values are collected in Table 1.

\subsection{Other components}

The optical receiver was modeled as a pin photodiode with a responsivity of $1 \mathrm{~A} / \mathrm{W}$ and with a thermal noise of $1.84375 * 10^{-22} \mathrm{~W} /$ $\mathrm{Hz}$, followed by a low pass Bessel electric filter to eliminate highfrequency noise.

The WDM multiplexer and demultiplexer elements are configured as optical Bessel filters, 2nd order, with a bandwidth of $10 \mathrm{GHz}$. They are fixed in all simulations.

\subsection{Simulated cases}

In the computer simulations four different systems have been analyzed based on the optical system of Fig. 1; Case A and Case B, using a DML-A in conjunction with SMF and NZ-DSF fibers, respectively. In Cases $C$ and $D$, the same scheme is used in substituting the DML-A by the DML-T.

Table 2 summarizes the different components used in each computer simulation.

Table 1

Simulation parameters for directly modulated lasers.

\begin{tabular}{lll}
\hline & DML-A & DML-T \\
\hline$\alpha$ & 2.2 & 5.6 \\
Volume active layer $\left(\mathrm{cm}^{3}\right) \mathrm{V}$ & $5.8 \times 10^{-12}$ & $1.1095 \times 10^{-10}$ \\
Gain compression coefficient $\left(\mathrm{cm}^{3}\right) \varepsilon$ & $5 \times 10^{-18}$ & \\
Quantum efficiency $\eta$ & 0.19 & \\
$\mathrm{k}(\mathrm{Hz} / \mathrm{W})$ & $28.7 \times 10^{12}$ & $1.5 \times 10^{12}$ \\
\hline
\end{tabular}


Table 2

Different configurations for the simulated system.

\begin{tabular}{lll}
\hline Case & DML & Optical fiber \\
\hline A & DML-A & SMF \\
B & DML-A & NZ-DSF \\
C & DML-T & SMF \\
D & DML-T & NZ-DSF \\
\hline
\end{tabular}

For each of these cases the system performance ( $Q$ factor) has been analyzed taken into account the three different modulation current types. The transmission system performance is often characterized by the Bit Error Rate (BER), which is required to be less than $10^{-15}$ for systems with a bit rate greater than $2.5 \mathrm{Gbit} / \mathrm{s}$. Experimental characterization of such systems is difficult since the direct measurement of BER takes considerable time and cost at such low BER value. For example, when a BER of $10^{-15}$ is target, a minimum measurement time of $27 \mathrm{~h}$ is required at $10 \mathrm{Gbit} / \mathrm{s}$ data rate. Another way of estimating the BER is to degrade the system performance by moving the receiver decision threshold value, as proposed in [6]. This technique has the additional advantage of giving an easy way of estimating the $Q$ factor of the system, which can be more easily modeled than the BER.

\section{Simulations and results}

For the simulation of the proposed system (Fig. 1), $100 \mathrm{~km}$ of optical fiber was used and, as already mentioned, only the results for the channel allocated at a wavelength of $1551 \mathrm{~nm}$ are presented. In all simulated cases (A, B, C and D) the laser was polarized with a bias current slightly below the threshold; that means no impact of the extinction ratio is considered. In all cases, a peak power for the "1" bit between 0.1 and $10 \mathrm{~mW}$ has been considered.

\section{1. $A$ and B cases: transmitter with dominant adiabatic chirp (DML-A)}

In a DML the optical output power of the laser is a function of modulation current, therefore, by using different expressions to define the current, different optical pulses are obtained. In Fig. 5(a) are shown the differences between the output pulses of the DML-A laser when the modulation current takes different forms: exponential, gaussian and sine.

The three pulses have the characteristic shape of the pulses generated by an adiabatic chirp dominated laser; when the current modulation type is gaussian, the laser takes much more time to achieve the carrier concentration threshold that produces a delay in the laser on-time and the leading edge of the pulse in relation to other types of modulation. This is one of the reasons why the system with a gaussian pulse has the worst quality.

In the case of an adiabatic chirp dominated laser, where the transient chirp has been completely "masked" by the adiabatic term, there is a shift between the frequency of the " 1 " and the " 0 " bits, having the frequency of the " 1 " greater than the frequency of the " 0 " (blue shift) [7]. Therefore, the result of the dispersion with the specific chirp characteristics is a high intensity spike at the front of the pulses and a trailing tail-end for transmission over a positive dispersion fiber, see Fig. 4b (Case A). Exactly the opposite effects will take place for transmission over a negative dispersion fiber, see Fig. 4c (Case B) [8]. In the case of an adiabatic chirp, dominated DML is the absolute value of the dispersion (and not its sign) which is playing the main role in the transmission performance [9]. Fig. 4(b) and (c) takes into account the simulation of a point to point optical link without any element of selectivity in wavelength; only the fiber introduces changes in the chirp of the transmitted pulse.

In Fig. 5 the maximum $Q$-factor, minimum BER of the system in Fig. 1 is shown. Variations on the DML optical power from 0.1 to (a) Pulse shape at the DML-A laser output.

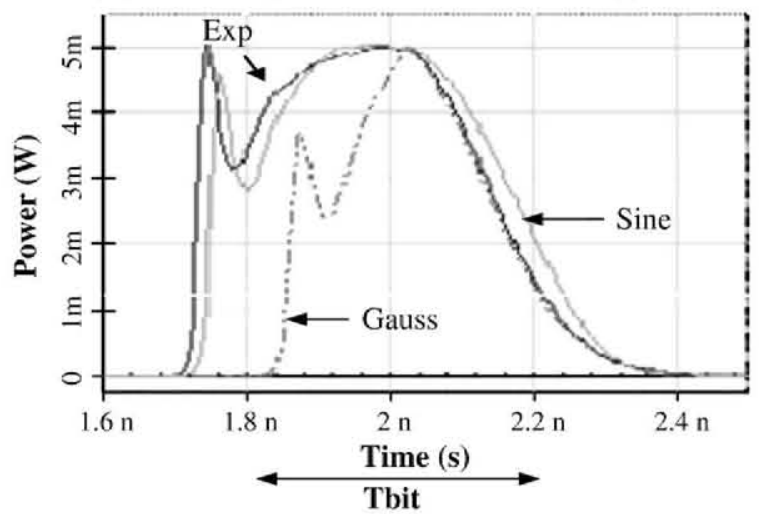

(b) Pulse shape after $100 \mathrm{~km}$ of SMF fiber (A case)

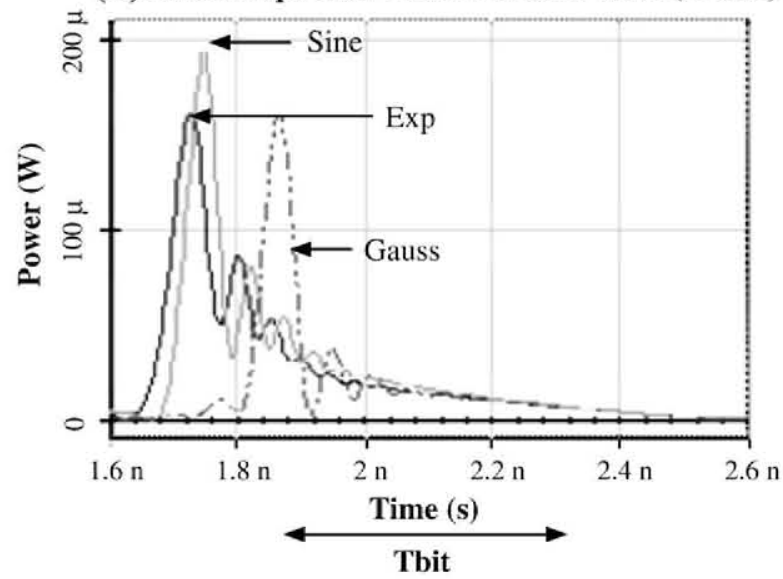

(c) Pulse shape after $100 \mathrm{~km}$ of NZ-DSF fiber (B case)

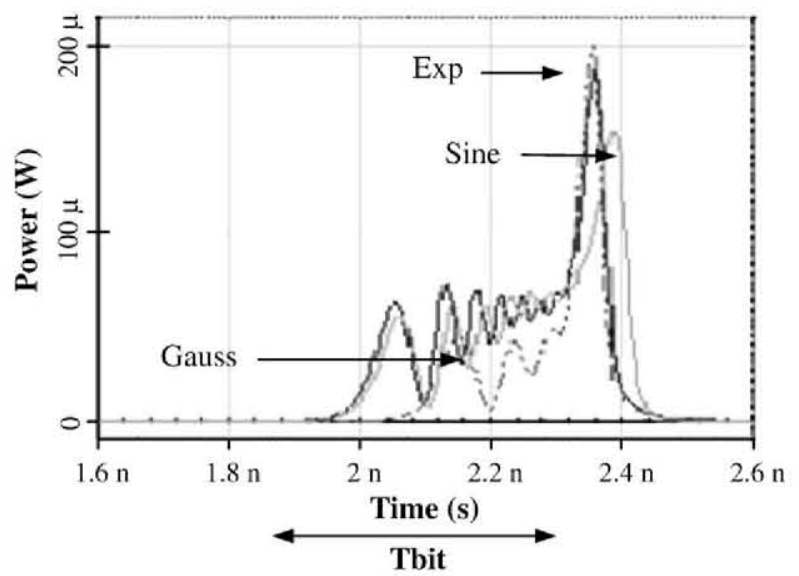

Fig. 4. Shape of the pulse for a DML-A laser with three types of modulation current: exponential, (thick dotted line), gaussian (thin dotted line) and sine (continuous line).

$10 \mathrm{~mW}$ for the three types of modulation current are analyzed for the (a) SMF fiber and the (b) NZ-DSF fiber. The graphs show that, regardless of the type of fiber used, the system has a maximum of quality for a given value of power, which agrees with the results obtained in previous works [10-12]. For low optical power levels, below the optimum power, the $Q$-factor increases with $P(t)$, mainly due to two reasons: one, because a larger amount of power reaches the detector and the performance of the system is improved, and, two, due to the interaction between the negative dispersion N-DSF fiber and the generated frequency of chirp, and for $P_{\text {opt }}$ there is the biggest compensation between them [13]. On the other hand, for a 
(a) SMF

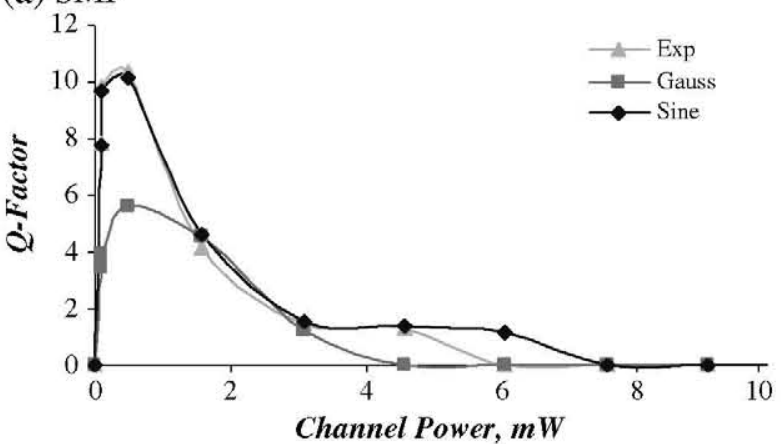

(b) NZ-DSF.

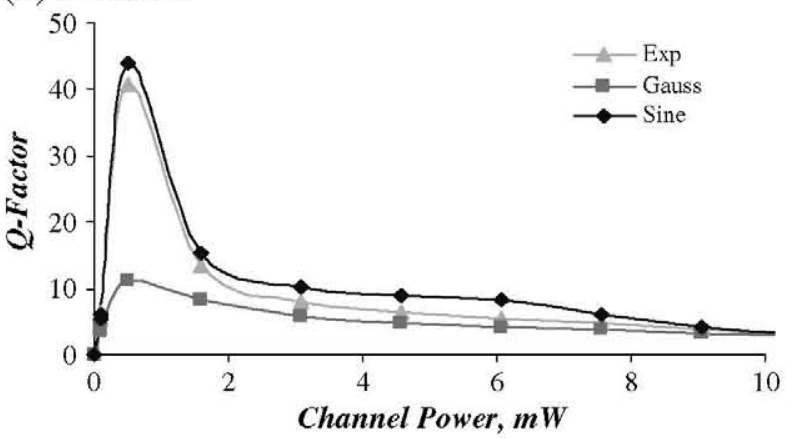

Fig. 5. Quality factor as a function of the DML-A output power on fibers with (a) positive dispersion coefficient and (b) negative dispersion coefficient.

$P_{\text {ch }}$ higher than the optimum power, the chirp increases with the power and it causes a greater frequency shift in the " 1 " and " 0 " transmission and linewidth broadening producing a bigger optical pulse deformation [8].

Moreover, in cases where lasers are used with a dominant adiabatic chirp (DML-A) the worst of the models is always obtained for the gaussian shape producing values of similar quality to the other two types.

Looking at Fig. 5(a) and (b) we can see that the system has a similar behaviour with the sine and exponential models and they are far from the gaussian model. These variations are due to the similarities/differences that exist in the form of pulses at the output of the laser when the different models are considered [see Fig. 4(a)].

If we compare the graphs of Fig. 5(a) and (b), the behaviour of the system depends on the type of fiber used because the chirp associated with the pulse transmitted depends on the value of the initial pulse chirp, the sign of the coefficient of dispersion $(s)$ and its absolute value $\left(\beta_{2}\right)$ [see (2)].

Fig. 6(a) and (b) shows the eye diagrams for a signal transmission over $100 \mathrm{~km}$ of SMF and NZ-DSF, respectively. The eye corresponding to the transmission over the SMF fiber is more distorted than the eye corresponding to the transmission over the NZ-DSF fiber because of the larger absolute value of the dispersion. The different dispersion sign will just affect the asymmetry of the eye diagram. All these changes in the pulse that reaches the receptor cause the differences between the $Q$ factors shown in Fig. 5(a) and (b).

\subsection{C and D cases: transmitter with a dominant transient chirp (DML-T)}

In the case of a transient chirp dominated laser DML-T, the leading edge of the pulse is blue shifted relative to the main portion of the pulse, while the tailing edge is red shifted (see the chirp pulse waveform in Fig. 7). The blue-shift chirped portion advances relative to the main portion of the pulses in the case of positive dispersion (a) SMF

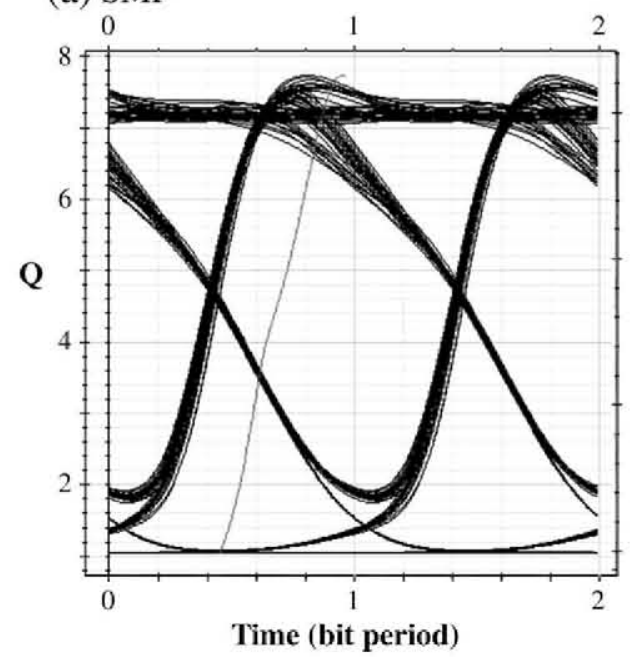

(b) N-DSF

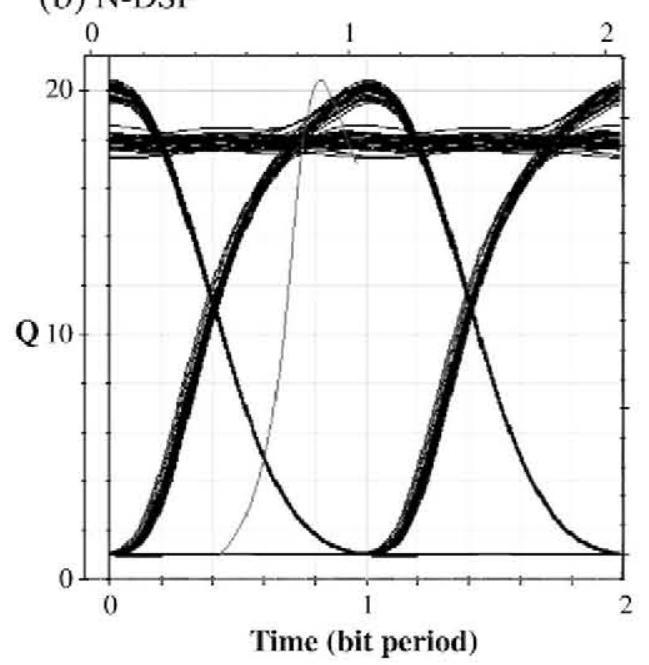

Fig. 6. Eye diagram for a DML-T laser with an optical output of $1 \mathrm{~mW}$ after $100 \mathrm{~km}$ of SMF (a) and NZ-DSF (b). A sine pulse is considered as a modulation current.

fibers. This effect produces a pulse spreading and as a consequence, an inter-symbol interference occurs.

Fig. 8 shows the $Q$ factor as a function of power for the three models studied. Fig. 8(a) corresponds to Case $\mathrm{C}$ and Fig. 8 (b) is dealing with Case D. In either case $C$ or D, again, they show the existence of an optimum optical power for a maximum $Q$. Also, in both cases, the minimum quality standard is achieved for the gaussian model. However, now the type of fiber used in the link determines the modulation current pulse shape for which the transmission is optimal.

Therefore, by using a negative dispersion fiber (Fig. 8b), the model with the exponential pulse is optimal for an output power of $2.6 \mathrm{~mW}$. In this case, the reduction in quality that the sine and gaussian pulse models have with respect to the exponential one is approximately $11 \%$ and $84 \%$, respectively. However, when using a fiber with a positive dispersion coefficient SMF (see Fig. 8a), the system behaviour is different. In this case, the modulation current with the sine shape provides a better performance, particularly in the case where the power of the laser is about $6 \mathrm{~mW}$.

This improvement of performance observed in Case C, with the sine modulation current, can be understood in terms of the interaction that is produced between the DML-T chirp with the sine modulation current and the fiber dispersion characteristic. As it is shown later, the 


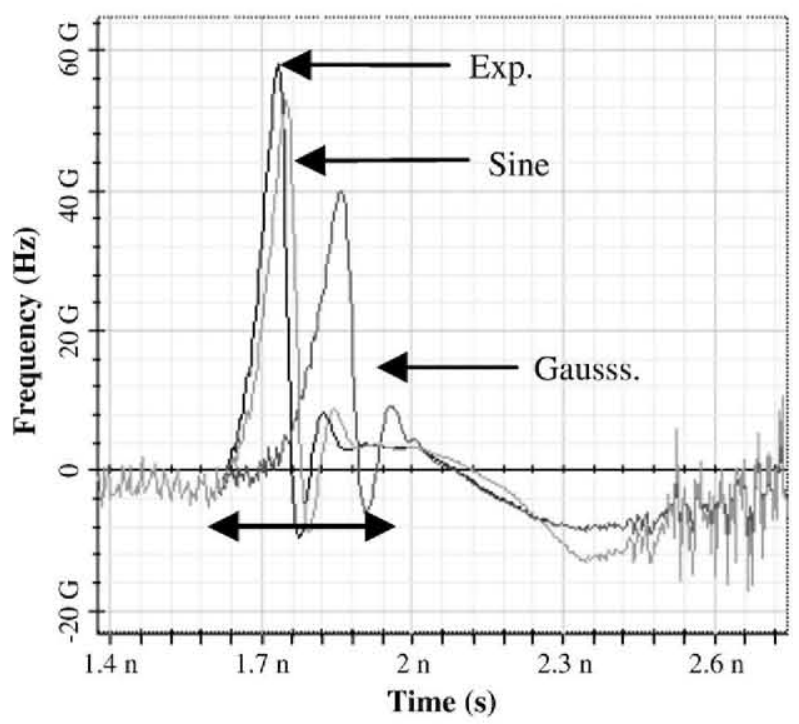

Fig. 7. Shape of the chirp pulse for a DML-T laser for three different types of current modulation: exponential, gaussian and sine.

phase accumulated by the transmitted optical pulse is practically constant in time.

When the chirped pulse travels along a fiber, it interacts with the fiber dispersion characteristics, causing a phase shifting in the pulse, which is a function of the sign and the absolute value of the dispersion coefficient [12]. Fig. 9 shows the eye diagram after $100 \mathrm{~km}$ of SMF (a) and NZ-DSF (b) by using a DML-T transmitter.

In both cases the modulation current was an electrical sine pulse. The eye corresponding to a transmission over the N-DSF fiber is more distorted than that corresponding to a transmission over the SMF fiber. This calls the attention because of the larger absolute value of the dispersion of the SMF fiber compared with the NZ-DSF fiber. On the other hand, the different dispersion sign will just affect the asymmetry of the eye diagram, as it is obvious from the results of Fig. $9(\mathrm{a})$ and (b).

\section{(a) SMF.}

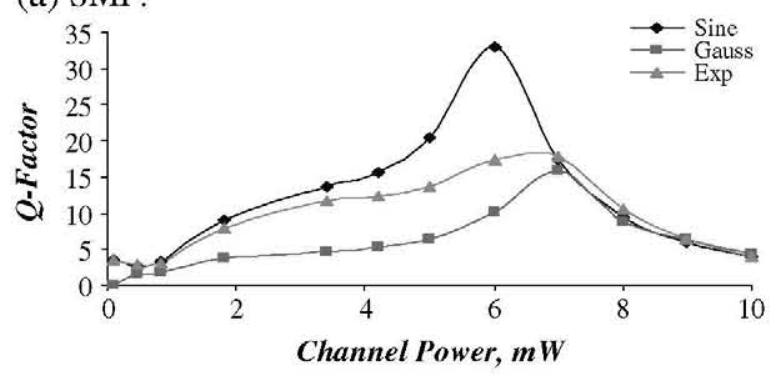

(b) NZ-NDF.

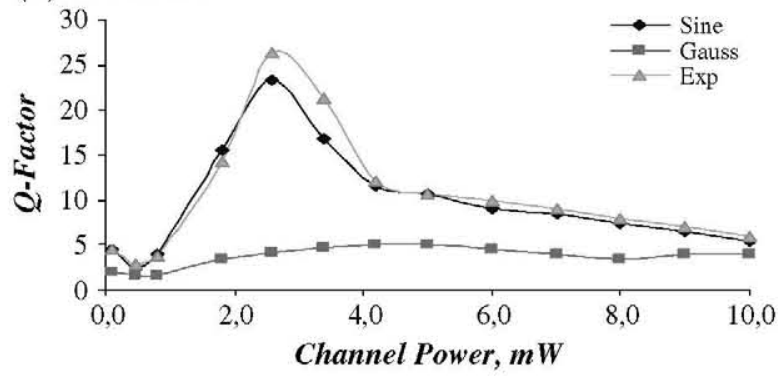

Fig. 8. Quality factor as a function of the DML-T output power on fibers with (a) positive dispersion coefficient and (b) negative dispersion coefficient. (a) SMF

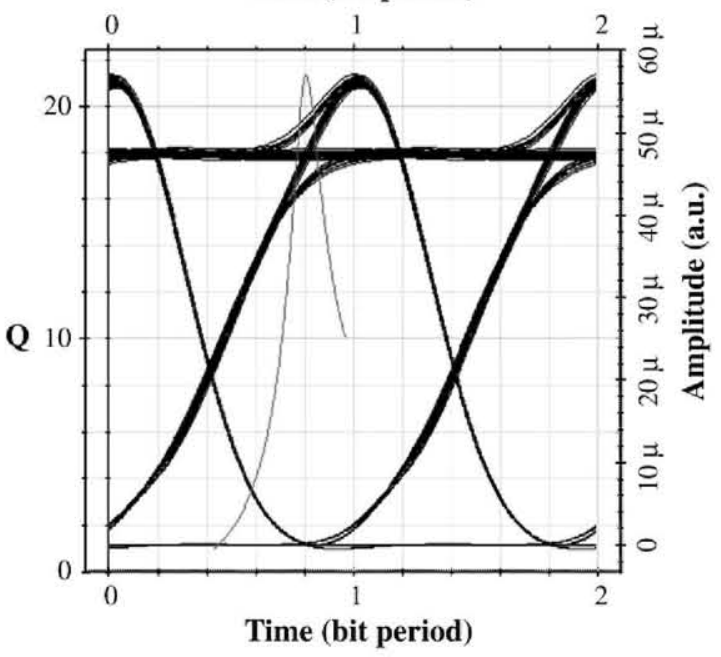

(b) N-DSF

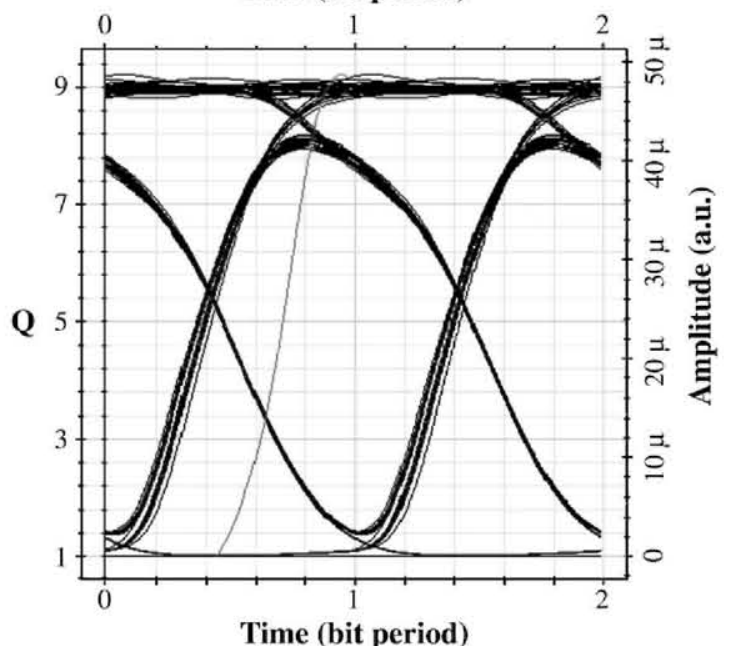

Fig. 9. Eye diagram for a DML-T laser with an optical output of $1 \mathrm{~mW}$ after $100 \mathrm{~km}$ of fiber. A sine pulse is considered as a modulation current

By the former simulations, it has been demonstrated that directly modulated systems using SMF fibers can reach similar quality factors (Q) as that of those using NZ-DSF fibers, by the utilization of a correct modulation current. It means, that, by an appropriated choice of the emitted optical power in the DML and also in the shape of the modulation current, the tolerance of the system at chirp can be optimized and its performance can be improved. This is an important conclusion due to the SMF fiber being the most installed, even operating at $1550 \mathrm{~nm}$.

To justify the difference between the behaviour of the sine model and the exponential and gaussian models, when using a DML-T laser with a positive dispersion coefficient fiber, Fig. 10 represents the accumulated phase variation over a sequence of 512 bits, for the different DML-T output power for the sine model after $100 \mathrm{~km}$ of SMF fiber. As it can be seen, there is a single value of power $(6 \mathrm{~mW})$ that produces a constant phase variation over time that would be the best choice for the transmission. For this value of power the phase variation is constant in time and the accumulated chirp is practically zero.

The phase variation is now represented for a sequence of 512 bits at the output of the DML-T laser with $6 \mathrm{~mW}$ [Fig. 11(a)] and before the 


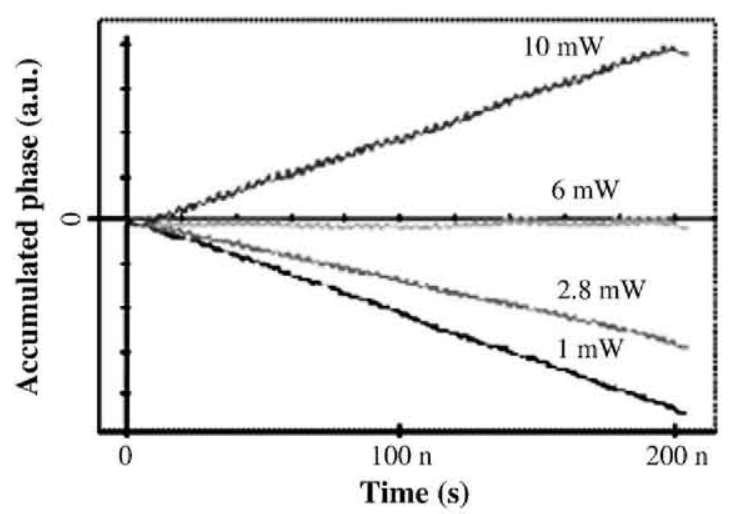

Fig. 10. Phase shifted (chirp) produced in a 512 bits sequence, for the sine model after $100 \mathrm{~km}$ of SMF fiber

bit stream reaches the receiver [Fig. 11(b)], for the exponential, sine and gaussian models. It is observed that the bit stream suffer different accumulated phase variations when crossing the SMF fiber and the filter components in the link (multiplexers, demultiplexers). The accumulated phase due to the sine pulse shape is practically constant, unlike that produced for exponential and gaussian pulses.

(a) At the output of the DML-T

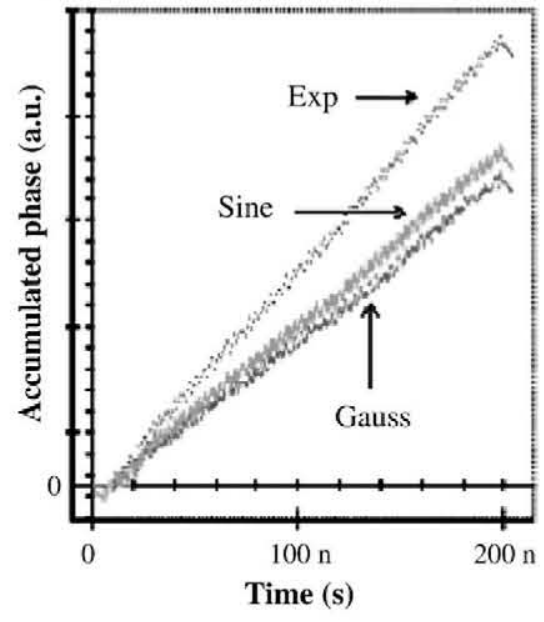

(b) At the output of the demultiplexer.

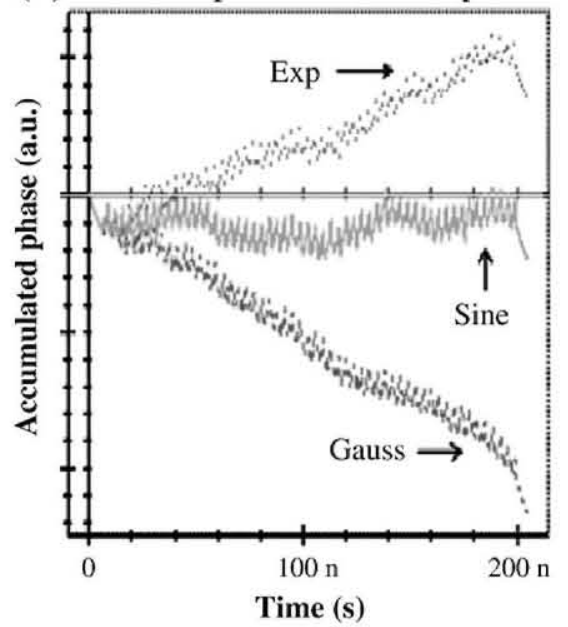

Fig. 11. Accumulated phase shifted (chirp) for a DML-T laser with $6 \mathrm{~mW}$ of optical power, 512 bits sequence, before and after $100 \mathrm{~km}$ of SMF fiber.
The high dispersion tolerance is mainly because of the phasecorrelative modulation among the adjacent bits via a precise control of the frequency chirp in the DML modulation. Therefore, based on $(1) \div(8)$ expressions of the chirp model (already including the drive current (DC) bias), we can design the parameters of the DMLs to offer a suitable chirp response for the generation of the phase correlation.

\subsection{Study of transmission performance over different lengths of SMF and NZ-DSF fibers}

In order to have a general idea about the behaviour of the systems in Fig. 1 with the link distance, a set of simulations was carried out varying the fiber length, $L$. As in the former cases, only results for a channel centred at $1551 \mathrm{~nm}$ are performed. The simulated transmitter was a DML-T similar to that used in the former sections. The output power is $P_{c h}=6 \mathrm{~mW}$ in the case of a SMF fiber [see Fig. 8(a)] and $P_{c h}=2.6 \mathrm{~mW}$ when a NZ-NDF fiber is used [see Fig. 8(b)]. These power values have been selected to obtain the $Q_{\max }$.

Fig. 12 represents the $Q$ factor versus the fiber length when a SMF fiber (a) and a NZ-NDF fiber (b) are used for sine, gaussian and exponential modulation currents, respectively. Since it was of waiting, the $Q_{\max }$ is reached for a fiber length of $100 \mathrm{~km}$ and a sine modulation current in the case of a SMF fiber. This is the length where the interaction between the accumulated dispersion on the fiber and the chirp generated in the DML is optimal. Nevertheless, for other fiber lengths, another choice can offer a better performance.

In the case of a NZ-DSF fiber, in Fig. 12(b), with a $\mathrm{P}_{c h}=2.6 \mathrm{~mW}$, the $Q_{\max }$ is obtained for lengths different from $100 \mathrm{~km}$, i.e, for a sine modulation current, $L=50 \mathrm{~km}$ [not exponential like in Fig. $8(\mathrm{~b})$ ]. Therefore, we can conclude that the maximum of the $Q$ factor depends on the modulation current shape, the link length, the optical power and the extinction ratio at the DML output.

\section{Conclusion}

In this study we have determined the dependence of the system performance of a directly modulated optical system with regard to the shape of the optical pulse transmitted across the fiber. It has been demonstrated that the worst behaviour corresponds, in all the studied

(a) SMF.

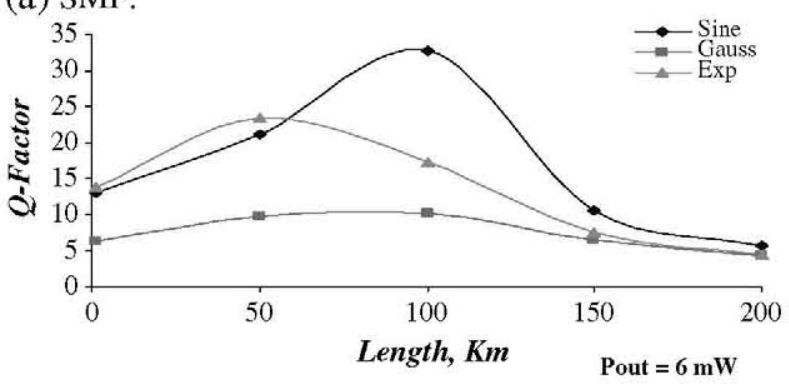

(b) NZ-NDF,

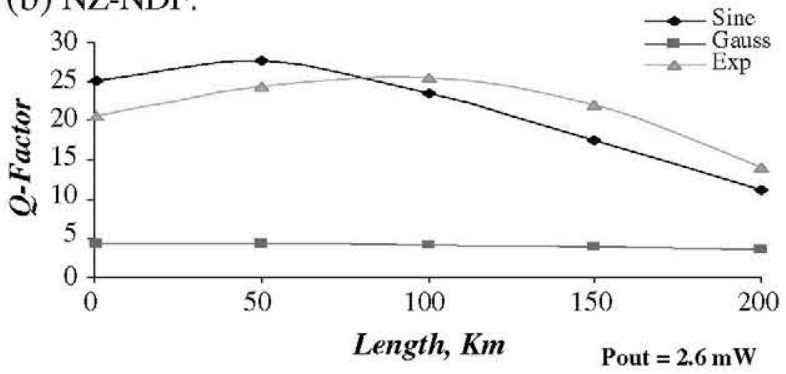

Fig. 12. Quality factor as a function of the link distance along fibers with (a) positive dispersion coefficient and (b) negative dispersion coefficient. 
cases, with a gaussian form of pulse and that, if the used laser has a transient dominant chirp behaviour, the chromatic dispersion coefficient sign of the fiber will be determinant at the moment of selecting the form of pulse that provides the best value of quality. For example, in the case in which a fiber is used with a high and positive dispersion value (SMF), the modulating current which presents a better performance is the sine form.

Taking into account that the gaussian model is the most restrictive as the quality refers, but is mathematically easy to handle, this model might be in use for realizing all the previous calculations in the design of optical communication systems and it might guarantee that any other model considered will overcome the minimal conditions of the quality needed.

We have also demonstrated that it is possible to improve the performance of an already installed system by modifying the shape of the electric current that modulates the laser. As the high dispersion tolerance is mainly because of the phase-correlative modulation among the adjacent bits, then, we can design the parameters of the DMLs, including the modulating current shape, to offer a suitable chirp response for the generation of the phase correlation. For SMF fibers (positive dispersion coefficient) this shape is senoidal and for NZ-NDF fibers (negative dispersion coefficient) the shape is exponential, when long-haul inter-office transport systems, corresponding to link distances of about $40-80 \mathrm{~km}$, are considered.

In summary, by appropriate combinations of DML transmitters (adiabatic or transient) and shape current, optical fiber systems can be achieved which are optimized in terms of dispersion and cost.

\section{Acknowledgements}

The authors gratefully acknowledge the support of the MICINN (Spain) through project TEC2010-18540 (ROADtoPON).

\section{References}

[1] Govind P. Agrawal, Fiber-Optic Communication System, A John Wiley \& Sons, INC Publication0-471-22114-7, 1997.

[2] Larry A. Coldren, Scott W. Corzine, Diode Lasers and Photonic Integrated Circuits, Wiley Series in Microwave and Optical Engineering, 1995.
[3] C.H. Henry, IEEE Journal of Quantum Electronics vol. QE-18 (Feb 1982).

[4] I. Tomkos, I. Roudas, A. Boskovic, N. Antoniades, R. Hesse, R. Vodhanel, "Measurements of Laser Rate Equation Parameters for Simulating the Performance of Directly Modulated $2.5 \mathrm{~Gb} / \mathrm{s}$ Metro Area Transmission Systems and Networks". Lasers and Electro-Optics Society, LEOS 2000, 2, IEEE, 2000, p. 692.

[5] Yu. Yanguang, Giuliani Guido, IEEE Photonics Technology Letters vol. 16 (No 4) (April 2004).

[6] N.S. Bergano, F.W. Kerfoot, C.R. Davidson, IEEE Photonics Technology Letters 5 (Mar. 1993) 304.

[7] A. Villafranca, J. Lasobras, R. Escorihuela, R. Alonso, I. Garcés, Proceedings of the OFC, Paper OWD4, San Diego (USA), Feb 2008.

[8] B.W. Hakki, Journal of Lightwave Technology vol. 10 (7) (Jul 1992) 964.

[9] I. Tomkos, D. Chowdhury, IEEE Journal on Selected Topics in Quantum Electronics vol. 7 ( $\mathrm{n}^{\circ} .3$ ) (May/Jun 2001)

[10] C. del Río, P. Horche, International Conference on Advances in Electronics and Micro-electronics, ENICS, 2008.

[11] P. Horche, Carmina del Río, Optical Fiber Technology vol. 14 (Issue 2) (April 2008) Pages 102.

[12] Suzuki Nobou, Journal of Lightwave Technology vol. 11 (№ 9) (Sep 1993).

[13] H.S. Jang, Y.G. Chung, Y.C. Chung, Optical Fiber Communication Conference, OFC Volume: 1 (2004)

Carmina del Río Campos received her Telecommunication Engineering degree from ETSIT (School for Telecommunication Engineering), Universidad Politécnica de Madrid (UPM) in 1996. Since 1997 she has made compatible her work in private companies (Via Digital and Banco Central Hispano) with lecturing in different Universities (Antonio de Nebrija, Carlos III). At present, she is realizing her Tesis Doctoral in the Universidad Politécnica de Madrid and she is working as a professor at the Universidad San Pablo CEU (Madrid), in the Telecomm. and Elect. Systems Dpt.

Paloma R. Horche received her Telecommunication Engineering degree in 1986 and her PhD degree in Communications in 1991, both from the ETSIT-School for Telecommunication Engineering, Universidad Politécnica de Madrid (UPM). Since 1990 she has been a member of the faculty of the ETSIT-UPM, currently as a Professor Titular de Universidad of Optical Communications in the Department of Photonic Technology. Her main research activities are in the area of Optical Signal Processing with special emphasis on the fields of Optical Communications and Systems. She is a member of OSA.

Alfredo Martin Minguez received his Telecommunication Engineering degree from ETSIT (School for Telecommunication Engineering), Universidad Politécnica de Madrid (UPM) in 1977 and the PhD degree in 2007. He has worked in Telettra España for twelve years developing optical transmitters and receivers. From 1992 he continued his work in Alcatel España, in FTTL. Optical Access Systems design. From 2004, he was working on holographic components for WDM transmission and, at present, on simulation of Quantum Dots Lasers, in the Dpt. of Photonic Technology. 